\title{
METACOGNITION AND CHEMICAL EDUCATION: AN EXPERIENCE IN TEACHING GEOMETRICAL ISOMERISM
}

\author{
Solange Locatelli, Agnaldo Arroio \\ Faculty of Education, University of Sao Paulo, Sao Paulo, \\ Brazil
}

\begin{abstract}
The purpose of the work presented is to analyze a way to introduce the concept of geometrical isomerism within a metacognitive perspective. This research was conducted with six students from high school, whose task was to build and distinguish geometric isomers with models made of plastic balls. The results indicate that this type of activity was favorable for expression of metacognition and metavisualization and, to some extent; this may have helped the students to identify the cis and trans isomers, allowing the construction of the initial concept of geometrical isomerism. Furthermore, there was the identification of other factors that affected the development of the task, in particular, the visual skills that seem very important for the student to become metavisual.
\end{abstract}

Key words: geometric isomerism, metacognition, metavisualization, visual ability visualization.

\section{Introduction}

The teaching of geometrical isomerism is relevant within the chemical content, as it allows you to approach the subject trans fats, including STSE perspective - Science, Technology, Society and Environment. This topic has been included in the teaching of chemistry: "Such curriculum have as main objective, to prepare students for citizenship and are characterized by an approach of scientific content in their social context" (Santos $\&$ Mortimer, 2002). The trans fat content, which may be developed from the concept of geometrical isomerism, has great technology and social implications have been sued, with the goal of food to be produced with lower content of such harmful fat, as was evidenced his harm people's health. As noted in a survey: "The primary metabolic effect of trans fatty acids in relation to coronary heart disease refers to its action hypercholesterolemic, making both high total cholesterol and low density lipoprotein (LDL-c), reducing high density lipoprotein (HDL-c) and this results in a significant increase in the ratio of $L D L$ c/HDL-c" (Chiara et al, 2002), in other words, trans fat increases levels of bad cholesterol (LDL) and lowers levels of good cholesterol (HDL). Chemically, called trans fat consists of trans fatty acids, of which there are compounds having the same molecular formula that these trans fatty acids, the fatty acid cis, and the structural difference is the geometry assumed by each. Thus, these geometric isomers (cis and trans) are defined as "compounds of the same type and number of atoms and chemical bonds with the same, but dif- 
ferent spatial arrangements, these isomers cannot be interconverted without breaking a chemical bond" (Chang, 1994).

Because of the importance in the study of this type of substance, the purpose of this work is the construction of the initial concept of isomerism geometric in a metacognitive perspective. The choice was made for the use of models of balls, because the concrete representations or pseudo-concrete seem to be more accessible than abstract representations (Ferk et al, 2003). Based on this manipulation, students will follow a script that will be able to realize that, despite apparently equal, the molecules differ in geometry. The class involves a strategy that motivates the expression of metacognition and metavisualization, high-level cognitive processes and fundamental to learning. Metacognition plays a central role in learning, because more than provide information, education should form a student who is able to ask questions and find answers, able to reflect on their knowledge and on how knowledge is produced, which is called metacognition (Parolo, Barbieri \& Chrobak, 2004). This is a relatively new term in the literature and has many definitions. According to Flavell (1976):

"Metacognition refers to one's knowledge concerning one's own cognitive process and products or anything related to them." In other words, metacognition refers to thinking about your thinking, being a high-level cognitive process. But not only that, metacognition also "refers, among other things, to the active monitoring and consequent regulation and orchestration of these processes in relation to the cognitive objects or data on which they bear." (Flavell, 1976, p. 232).

Indeed, metacognitive strategies can help to monitor the construction of knowledge, redirecting when necessary. An example that illustrates this was a survey of three students, in which it was proposed to solve a problem of chemistry. The first student, graduated in chemistry, tried to solve the exercise, but failed to reach the expected result. At the same time, a pair of students from 1st year of undergraduate chemistry course had the same doubts that the first student, but could reflect on it, expressing metacognition and thus reached the correct result of the problem (Rickey \& Stacy, 2000).

Reflecting on the proposed activity for teaching isomerism geometric, balls' models were used so that students could manipulate them and visually perceive differences or similarities between the models made of molecules. There was encouragement to think about a view, namely, to express metavisualization, a term suggested by Gilbert (2005). Aiming to understand metavisualization, primarily, due to conceptualize visualization that according to the context also takes on different meanings. In this way, we have two different meanings in science education: it can be used with a verb (to visualize) or as a noun (visualization) (Gilbert, Reiner \& Nakleh, 2008). A model of balls may be a visualization, but the internal form as the individual interprets the information, is the action of visualize (Figure 1). Thus, "to take advantage of visualizations, we argue that students need cognitive understanding of the phenomena as well as metacognitive skills to guide their own learning" (Chiu \& Linn, 2012).

Thus, it is understood that metacognition takes metavisualization name, which means, in this case, to rethink the image created with the student models, seeking to identify the isomeric substances. 


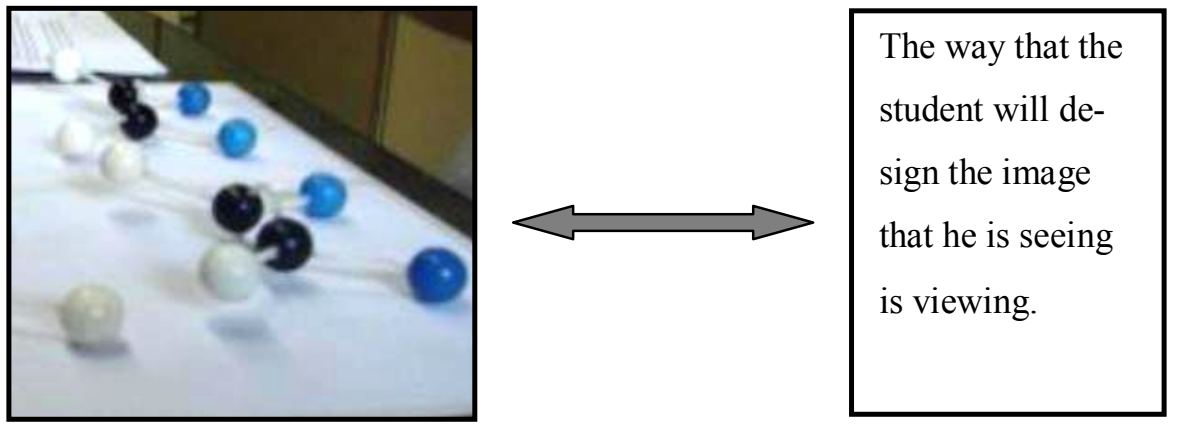

Figure 1: Visualization (left) $\mathrm{x}$ to visualize (right).

Thus, it is very important that the placement of:

"The visualization is a crucial element in learning chemistry, as it is and produces models, so it is necessary for the student to be metacognitive in relation to visualization, namely, to develop the ability to metavisualization to appropriate the models used to explain different contents in chemistry." (Locatelli; Ferreira \& Arroio, 2010, p. 81)

\section{Methodology of Research}

The research was conducted with six students from the 16 years old, 2nd year of high school, organized into four groups, with two students (A1 and A4) that performed the activity individually - groups 1 (A1) and 2 (A4) - and four students (A5, A6, A7 e A8) formed two doubles - groups 3 (A5 and A6) and 4 (A7 and A8). They had studied isomerism plane, but not the spacial and the activity consisted in building models of balls and the recognition or not of the geometric isomers relative to 1,2-dichloroethene.

Precisely the focus of the research was to understand how students think to perform the activity, to what extent was the manifestation of metavisualization of their reasoning. For this purpose, the instruction given to pupils is that the activity would be audio recorded, and that they should talk aloud about whatever they were thinking, without exception, namely, the orientation was speak aloud whatever they were thinking. As already explained, the purpose was to examine whether there was demonstration of metacognition or metavisualization within the students talking during the development of the activity, and to what extent this could help in the identification of geometric isomers. Although it is difficult to minimize the influence of the students were being watched, they were trained by repeatedly performing other activities, which certainly helped in this goal.

Thinking aloud protocols is a method that has been used in research to study metacognition (Veenman et al, 1993), since "methods employed are chosen according to the methodology and the research paradigms in which the study is embedded" (Anderson, Nashon \& Thomas, 2009). Initially, the researcher followed and watched the process. The concern was not whether the students would hit or miss, but if they thought about it, exposing his previous ideas freely, thus enabling the construction of the concept from them and interaction with the material.

When the activity was almost finished, the researcher did some interference when necessary, to make them think about what they had done - directing one more time for the 
reflective exercise - so that they might realize that two distinct groups existed in the case, the isomers. Later, in the next class, the researcher and also the class teacher began the lesson by bringing the contributions of students and were collectively building the concept of geometrical isomerism and, as a result, a deeper study of the subject was made.

\section{Description of proposed activity for data collection}

Started up from four pieces of molecules models, made with balls (Figure 2) which correspond to four molecules incomplete - the proposal was that they should complement this model with other dots representing atoms, considering the blue (chlorine) and white (hydrogen) to form the 1,2-dichloroethene:

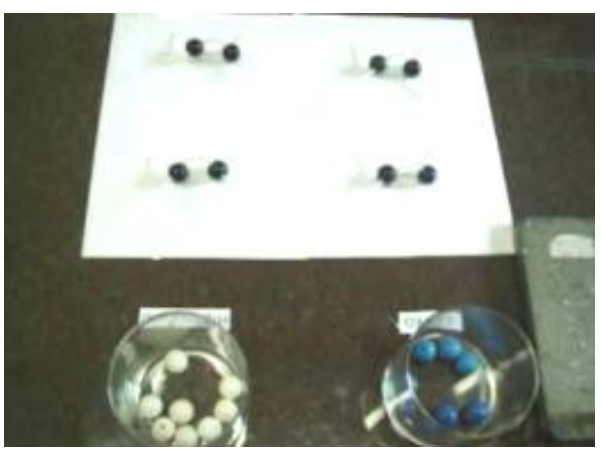

Figure 2: Four pre-molecules models with two carbon atoms joined by a double bond and four connections to be completed with hydrogen and chlorine.

Build four (4) representations of the mol cule of the compound 1,2-dichloro-ethane. A range them differently modifying the position the atoms of chlorine and hydrogen. Now, analy ing representations of molecules, do you think $\mathrm{t}$ ] molecules are the same or different?

That is, you just build one or two types molecules? Discuss with your partner.

The hypothesis was that students could build models and concluded that there were two distinct groups of molecules, with or without mediation by the researcher. In addition, another objective was to observe whether or not there would be a expression of metavisualization and to what extent this could help them identify the different isomers and finally, possible factors that interfere in the process.

\section{Results of Research}

Results Group 1 - A1 Student: He took two minutes to accomplish this task and apparently had no difficulty in implementation. She began building a cis representation of the molecule of the compound and quickly built the second molecule, this time being trans. Following, he set up another representation of the trans molecule, and A1 goes on to explain and show that, spatially, molecules represent the same thing:: "Now ... I'm putting in a different order, just as in the case here, they are representing the same molecule ... I'm creating now, because it would spatially the same substance (it shows the cross chlorines) because of double that just separating..." He does the same thing and goes on to explain with another cis molecule, justifying spatially: "In two dimensions they might look different, but in this case, spatially they are the same molecules."

And rotating the molecule which had the chlorines down, it shows two representations of molecules with cis chlorines upwards (Figure 3): 


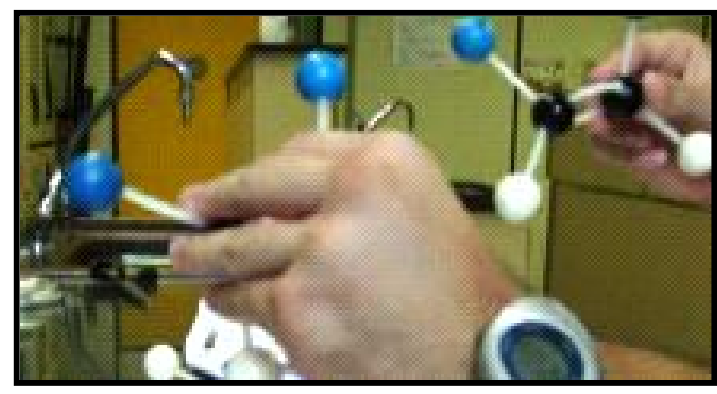

Figure 3: A1 student showing the two representations of the cis molecule.
Result Group 2 - A4 student: She began building a representation of the trans molecule, followed by a cis. Right now stopped to think a little, stared for a few seconds the two molecules that had done: "Now here... let me see...to be different ... the two chlorine and two hydrogen and ... that other hydrogen here" When trying to build the other cis molecule, mingled and build a molecule of a different compound, 1,1-dichloroethene. She for

herself is questioned and fixes, realizing the mistake and redoing the molecule, this time riding correctly to another representation of the cis molecule. The student being questioned if the four molecules built would be the same hesitates a bit and responds: "well... I'm thinking...I think the molecules are equal because, for example, if I turn that well, stays the same..." She does not show at that time, she could distinguish between the two groups of molecules. Hesitates a bit, looks back and turns the molecules and she can see that two are equal, showing two cis molecules. Following, she does the same thing for the trans molecules, appearing this time she has realized that there are two groups of molecules (Figure 4).

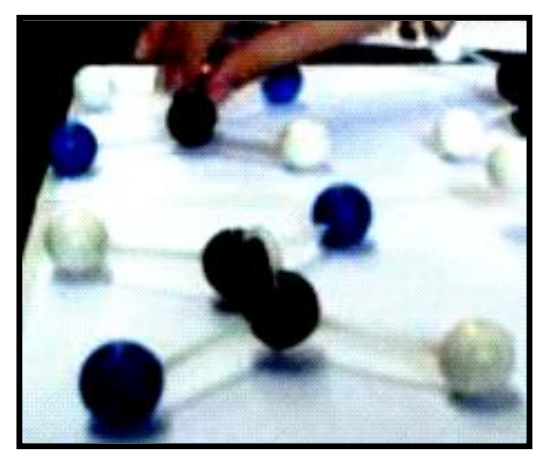

Figure 4: Equal Representation of the trans molecule.

Then the researcher asks the student explain spatially on molecules made. The student shows with her hands and rotating molecules, states recognize the existence of two distinct sets of molecules. Results of Group 3 - A5 and A6 Students: The pair interacts well and begins building a cis molecule, one trans and another cis molecule. At this point, A6 questions saying "ah, it's all the same to me." With hands, A5 shows that a representation of cis molecule is equal to another cis molecule, justifying spatially "Well .. So ... this model with this model are the same because this one can rotate, but that other (points to the trans) I'm not sure. "They finish building the other trans, it seems there was a bit of doubt and hesitation, but A5 also identifies the trans molecules, separating the four molecules into two distinct groups. A6 seems to disagree or do not understand right: "So, all molecules represent the same substance." At this point the researcher intervenes and asks, "But tell me one thing, how many substances do you have there?" At the same time, A6 responds three and A5 responds two.

The researcher requests them to show, spatially, molecules, and A5 seems to understand, spatially, that there are two groups of molecules, but it is not clear to A6. When A5 starts explaining, shows the two cis with his hands. A6 smiling intervenes and says " Oh yeah, I thought they were the elements, carbon, hydrogen and chlorine ... three!." Thereafter A6 also gets to demonstrate that thinks spatially and apparently it seems that 
the obstacle was being understood what was being asked because she thought they were 3 elements. Seeing the demonstration of molecules by A5, she realizes the mistake. This indicates that A6 may have confused element or molecule and perhaps she did not understand the question nor had difficulty thinking visually. Now, the student A6 quickly shows and explains spatially trans molecules (Figure 5):

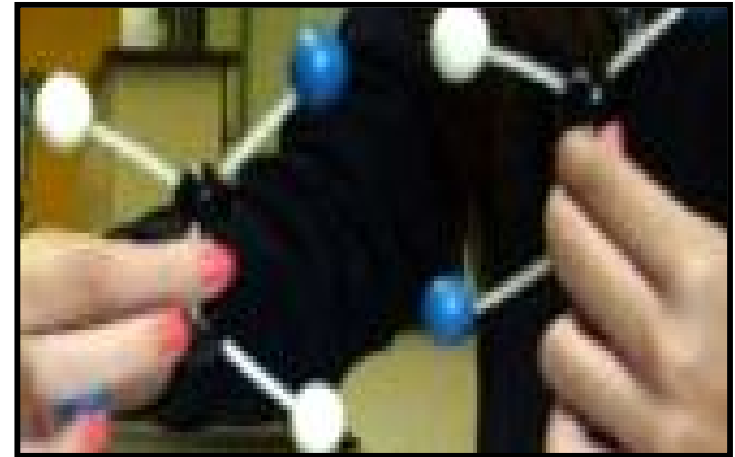

Figure 5: A6 shows the spatial representations of trans molecules.

Result Group 4 - A7 and A8 students. First, the students started making the build of two cis molecules. As a A8 student was not expressing his thoughts and showing much hesitation, the researcher asked A8 what she was thinking at that moment and she said: "Ah ... I'm thinking like her, the molecules will have... there is no difference between them, provided they have two chlorine and two hydrogen atoms, whatever the side ... understand?" At this point, A8

built erroneously the third molecule, placing the two chlorines on the same carbon. What may be an indication of not having understood that they were two chlorines forming the molecule, however in different carbons. Right now, the student A7 was finalizing the build of the other trans molecule, and the researcher interrupted asking: "Does this molecule, built by A8 is correct?" A8 did not answer, looked at the molecule that had just done. A7 quickly dismantled this molecule and concocted another trans molecule, demonstrating their understanding of how to make the compounds 1,2-dichloroethene. A8 seemed not quite understand the error, but eventually agreed. Both A7 and A8 conclude that all molecules are identical.

At this point the researcher asked for clarification: "Do you think that four molecules are alike?" They respond again that yes, in principle just looking for molecules made up, soon began to move, rotate, look on one side, the other, they seem in doubt, but ratified the molecules were equal, seeming not succeeded to identify spatial differences, explaining that carbon is always connected to a chlorine and a hydrogen, then it is the same thing. As the students seemed secure in his statement, the researcher has released another question to allow the conflict of ideas: "Now, if I told you that we have two substances there, are you able to show me the same molecules?" A7 surprised, caught the molecules in hand, was rotating them at length and answered, "yes, these two are equal (both cis)." Researcher seeks to show that spatially. The student A8 only observed, demonstrating not be understood. A7 shows that the two cis molecules are identical, showing that. And pointing to the other two trans molecules says: "And now these two (points to the trans molecules) are equal." But even with the trans molecules, the students outline very difficult to show that spatially are equal, spinning, rotating and reaching the wrong place and yet, saying the representations were equal (Figure 6): 


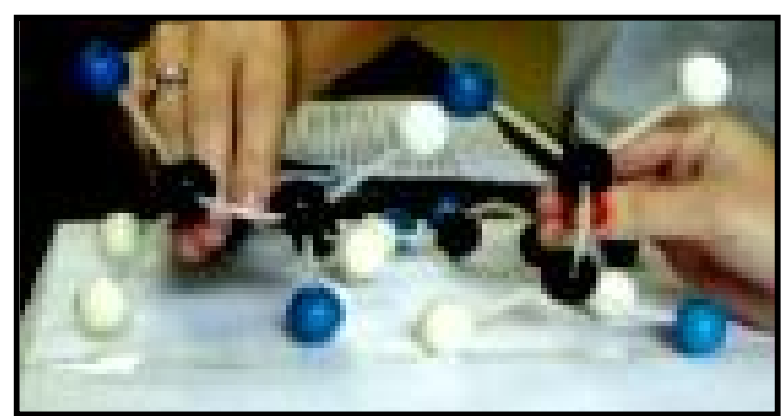

The researcher said, "But it is different from the way you are holding ..." Then finally, mediated by the researcher, they position the molecules spatially in order to show that they are actually equal (Figure 7).

Figure 6: Students position representations of trans molecules spatially and claim that they are the same, but cannot show it.

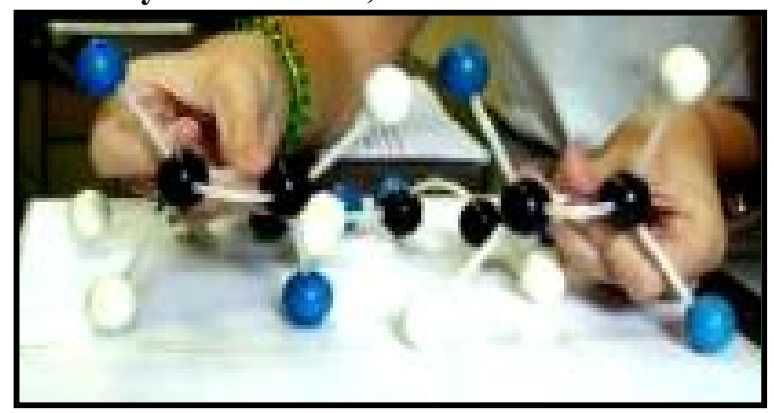

Figure 7: Students spatially positioned and properly representations of trans molecules after mediation by the researcher.

\section{Discussion}

With all the observation and analysis conducted, it can be stated that there is evidence that the A1 student has thought spatially in this activity, since at no time he showed hesitation, managing to finish quickly and spatially display all models. Also demonstrated the ability to distinguish between two groups of different molecules with spatial thinking, and these signs observed may indicate that A1 has thought metavisually and maybe that has helped in the development of the activity. Very similar occurred with the A4 pupil, with the difference that it appears that the expression of metavisualization may have helped her to identify two groups of molecules which, in principle, it was not identified. But there was also evidence of metavisual reasoning, just when she rethought looking for models and redirected his reasoning, leading to a correct identification.

Furthermore, A5 student gave evidence of spatial reasoning, as demonstrated facility in completing the task of this activity, having no difficulty in recognizing both groups of molecules spatially and explain. As regards A6 student, after clarification of conceptual error, between element and molecules, she seems that also gave evidence of spatial reasoning, but the question was whether A6 does have visual ability, since there was no clear evidence that this activity. This may also suggest that an obstacle in the reasoning of the metavisual can be a conceptual error or even the understanding of the task to be performed or, perhaps, A6 may have understood the spatial differences after an expla- 
nation of the student A5, indicating in this case, a difficulty in thinking spatially. Nevertheless, A7 and A8 seemed to have great difficulty in demonstrating visual ability to distinguish different molecules and recognize molecules that are equal. After much prodding, A7 seems to have given indications that she has, to some extent, this ability since managed to separate the cis molecules, but both A7 and A8 had much difficulty in showing that spatially trans molecules are equal. This could be a very big obstacle in the ability of metavisualization, because if they cannot think spatially, it could compromise the metavisual thought (Locatelli, 2011).

The research sought to answer whether, in this type of activity could have or not the expression of metavisualization and, to what extent, this could help students identify the different isomers and finally, still possible factors that might interfere with this process. According to Rickey \& Stacy (2000) dual activities may promote the manifestation of metacognition and self-regulation that can help in the process and this is consistent with what was observed with almost all students. It was observed that is possible to promote the expression of metavisualization, and it had evidence of this occurrence with 5 of 6 students, unless the student A8, because she did not show at any time, that she were thinking metavisually, though, she might have thought and not shown. On the other hand, the activity, as it was proposed, allows us to observe, to some extent, the visual ability of students. According to Wu \& Shah (2004), the students demonstrate difficulty in interpreting the visualizations, both internal and external and these visual skills are fundamental in chemistry. However, A1, A4 and A5 students seemed to demonstrate easiness in manipulation of models, leading to the conclusion that it is possible that they have a good visual ability. A6 and A7 seem to have this ability to some extent, but showed more difficulty compared to A1, A4 and A5students. Finally, it was not possible to verify whether the student A8 has this visual ability or not, because at no time, it was observed, and this is in agreement with what Wu \& Shah (2004) pointed. Another point that can be raised is that perhaps the A8 student has not understood the convention used in the representations, which according to Gilbert (2005) is a condition for the student to think metavisually.

\section{Conclusions}

Thus, the conclusion seems to indicate that the metavisualization may contribute positively in learning, and this type of activity proposed for the initial study of geometrical isomerism had its positive contribution and facilitator. However, other factors possibly be aggregated to this building, described in the following. The visual nature and also motivating of the activity, as students pointed out, may have been a factor in the expression of metavisualization. Visual skills appear to be an essential factor for the metavisualization is expressed as visual thinking skills refers to the assumption that the student has, at least, to some extent this ability, so you can think about it (metavisual).

The previous ideas can become a facilitator or an epistemological obstacle, prompting A6 student being confused to identify, because she thought it was to identify the types of atoms and not molecules. Likewise, it is essential to understand the representations in the proceedings as may have been observed with the student A8. Finally, the position of mediator researcher in pursuit of knowledge building and collaborative work may have helped, and really, to groups 3 and 4 . All these factors influenced the development of the activity. Hence, there is still much scope there should be researched regarding 
metacognition and metavisualization in science education, particularly in the teaching of chemistry, which aims to be a matter of great possibility and need for future research to further clarification.

\section{References}

Anderson, D., Nashon, S., \& Thomas, G. P. (2009). Evolution of research methods for probing and understanding metacognition. Research in Science Education, 39, 181-195.

Chang, R. (1994). Química (5.a Ed.). Portugal: McGraw-Hill.

Chiara, V. L., Silva, R., Jorge, R., \& Brasil, A. P. (2002). Ácidos graxos trans: doenças cardiovasculares e saúde materno-infantil. Revista Nutrição, 15 (3), 341-349.

Chiu, J. L., \& Linn, M. C. (2012). The role of self-monitoring in learning chemistry with dynamic visualizations. Metacognition in Science Education: trends in current research, 40, 133-163.

Ferk, V., Vrtacnik, M., Blejec, A., \& Gril, A. (2003). Student's understanding of molecular structure representations. International Journal of Science Education, 25 (10), $1227-$ 1245.

Flavell, J. H. (1976). Metacognitive aspects of problem solving. Em L. B. Resnick (Orgs), The nature of intelligence. Hillsdale, N.Y.: Erlbau, 231-235.

Gilbert, J. K. (2005). Visualization: A Metacognitive Skill in Science and Science Education. In J. K. Gilbert (Eds.) Visualization in Science Education. Holland: Springer, 9-27.

Gilbert, J. K., Reiner, M., \& Nakleh, M. (2008). Introduction. In J. K. Gilbert et al (Eds.) Visualization: Theory and Practice in Science Education, Holland: Springer, 1-24.

Locatelli, S., Ferreira, C., \& Arroio, A. (2010). Metavisualization: an important skill in the learning chemistry. Problems of Education in the $21^{\text {st }}$ Century, 24, 75-83.

Locatelli, S. (2011). A análise da manifestação de elementos de metavisualização na aprendizagem de Química. 155p. Dissertação - Universidade de São Paulo, São Paulo.

Parolo, M. E., Barbieri, L. M., \& Chrobak, R. (2004). La metacognición y el mejoramiento de la enseñanza de química universitaria. Enseñanza de las ciencias, 22 (1), 79-92.

Rickey, D., \& Stacy, A. M. (2000). The role of metacognition in learning chemistry. Journal of Chemical Education, 77 (7), 915-920.

Santos, W. L. P., \& Mortimer, E. F. (2002). Uma análise de pressupostos teóricos da abordagem C-T-S (Ciência - Tecnologia - Sociedade) no contexto da educação brasileira. Ensaio, Pesquisa em Educação em Ciências, 2 (2), 1-23.

Veenman, M. V. J., Prins, F. J., \& Verheij, J. (2003). Learning styles: Self-reports versus thinking aloud measures. British Journal of Educational Psychology, 73, 357-372.

$\mathrm{Wu}, \mathrm{H}$., \& Shah, P. (2004). Exploring visuospatial thinking in chemistry learning. Science Education, 88, 465-492.

Received 02 February 2013; May 24 February 2013

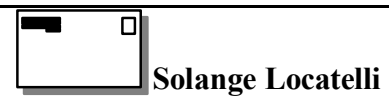

Chemistry Teacher, Master in Science Education, Faculty of Education, University of São Paulo, São

Paulo, Brazil.

E-mail: solangewl@hotmail.com

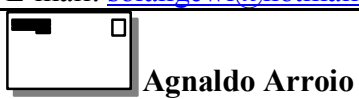

$\mathrm{PhD}$, Associate Professor, University of Sao Paulo, Faculty of Education, Av. da Universidade 308, bloco A, sala 109., Butantã, 05508-040 São Paulo - SP - Brazil

E-mail: agnaldoarroio@yahoo.com

Website: http: / / usp-br.academia.edu / AgnaldoArroio 